\title{
Retraction Note: Analysis of extreme programming
}

\author{
Ya Feng $^{1 *}$, Dongliang $\mathrm{Cui}^{2}$, Hui $\mathrm{Li}^{1}$, Ying Zhao ${ }^{1}$ and Jia Liu ${ }^{1}$ \\ ${ }^{1}$ Atmospheric Sounding Technology Assurance Center of Shanxi Province, Taiyuan, China \\ ${ }^{2}$ Early Warning Center of Shanxi Province, Taiyuan, China
}

Published online: 04 March 2020

Retracted article: MATEC Web of Conferences 309, 02016 (2020)

https://doi.org/10.1051/matecconf/202030902016

We take a zero tolerance to any situation where fraudulent research is published in our journals. As a result, this article has been retracted by the Publisher because it is suspected to be a nonsensical publication computer-generated by the program SCIgen. 